\title{
A Review Study on Amplification of X-Ray Free Electron Laser Pulse in Plasma
}

\author{
Ashutosh Sharma \\ Department of Education, University of Lucknow, Lucknow, India \\ Email: a_physics2001@yahoo.com
}

Received September 29, 2012; revised November 1, 2012; accepted November 9, 2012

\begin{abstract}
In view of X-ray Free Electron Laser (XFEL) intensity prospects, we reviewed the past and recent work in view of amplification of powerful XFEL laser pulse to achieve intensity in the regime of high field science. We report here some of the relevant work investigated in this field and predicted further scalings and possibilities for XFEL pulse amplification.
\end{abstract}

Keywords: X-Ray Free Electron Laser; Raman Amplification; Laser-Plasma Interaction

\section{Introduction}

The recent advent and active operation of X-ray free electron lasers (FEL) enabled this machine to capture the image of atoms and molecules in motion. These machines are routinely running experimental programme to study the properties of condensed matter, nanosystem, molecular and atomic processes, biological systems and chemistry. The first soft x-ray FEL facility, FLASH at DESY, has been in operation for users since 2005 [1]. The first hard x-ray FEL facility, the Linac Coherent Light Source at SLAC [2], became operational in 2009. More recently, the SACLA at SPring-8 [3] started its user program beginning in 2012. A series of experiments conducted at the Linac Coherent Light Source (LCLS) [2] have shown how ultraintense and ultrashort X-rays interact with various systems: light atom $(\mathrm{Ne})[4,5]$, molecule $\left(\mathrm{N}_{2}\right)$ [6-8], and solid (Al) [9]. In this soft and hard $\mathrm{X}$-ray wavelength range FELs are the only source that can produce coherent photons, with very high peak and average power and brightness higher by any orders of magnitude than for any other X-ray source and a number of photons per coherent volume of the order of $10^{9}$ or larger. There are high-field science applications of the X-ray FEL, taking advantage of not only the high energy and coherency of photons, but also their extreme high fields, which interact with matter in unique way. If the amplification of XFEL laser pulse can be practised; there will be great exposure of these intense X-rays to explore the issues of fundamental physics. In the spectral regime of optical wavelength high field science regime begins to occur at intensities of $10^{14}-10^{15} \mathrm{~W} / \mathrm{cm}^{2}$, where some new atomic physics phenomena have been observed. At relativistic intensity $10^{18} \mathrm{~W} / \mathrm{cm}^{2}$, relativistic effects fully enter into dynamics. The frequency of the XFEL pulse is four orders of magnitude higher than that of an optical laser, the corresponding intensities would be $10^{22}-10^{23}$ $\mathrm{W} / \mathrm{cm}^{2}$ and $10^{26} \mathrm{~W} / \mathrm{cm}^{2}$, respectively. However the anticipated intensity at LCLS, is $10^{17} \mathrm{~W} / \mathrm{cm}^{2}$ which is much less than the relativistic intensity at X-ray wavelength.

In view of XFEL intensity prospects as mentioned above, we reviewed the past and recent work in view of amplification of powerful XFEL laser pulse to achieve intensity in the regime of high field science. We report here some of the relevant work investigated in this field and predicted further scalings and possibilities.

Several schemes [10-12] are proposed for significant reduction of duration of powerful X-ray pulses to $0.3-1$ fs. Available optical techniques [13-15] are capable of $\mathrm{X}$-ray focusing to sub $50-100 \mathrm{~nm}$ spot size. There are proposals $[16,17]$ on focusing of X-rays to few nm spot size. However these focusing techniques can withstand only low intensity X-ray pulse and might not directly applicable to powerful LCLS X-ray pulse for which focusing appears to be more challenging. By means of backward Raman amplification [18] in plasmas one may compress the X-ray pulse to electron plasma wave period $\left(t_{0}=2 \pi / \omega_{e}\right)$ where $\omega_{e}=\left(4 \pi n_{e}^{2} / m_{e}\right)^{1 / 2}$ and $n_{e}$ is electron density in plasma. For electron density $=10^{26}$ $\mathrm{cm}^{-3}, t_{0}=10 \mathrm{asec}$. Longitudinal compression of $2 \mathrm{~mJ}$ $\mathrm{X}$-ray pulse to $10 \mathrm{asec}$ would produce the power 200 TW. The intensity of such X-ray pulse focused to spot diameter of $0.5 \mathrm{~nm}$ would be of the order $10^{28} \mathrm{~W} / \mathrm{cm}^{2}$. Since the electron density of the order of $10^{26} \mathrm{~cm}^{-3}$ can be accessed only at NIF facility and there is no plan to merge the NIF facility with LCLS to practise the ampli- 
fication of X-ray laser. Thats why it is not clear that the BRA mechanism can be practised in the X-ray regime or not. A simple scalings based on the BRA in optical regime is not sufficient to predict the efficient BRA in $\mathrm{X}$-regime because competing effects (inverse bremsstrahlung of the pump, collision and Landau damping of plasma wave, plasma heating etc.) might narrow or close the parameter window for efficient BRA operation. To realize the Raman amplification of X-ray laser pulses, requires the high plasma density (near to solid state density $10^{26} \mathrm{~cm}^{-3}$ ). For plasma densities below $10^{19} \mathrm{~cm}^{-3}$, ionization and electron-ion collisions do not often occurs during the pump-plasma interaction time to be of much relevance. When we proceed to practice the Raman amplification of X-rays beam which needs plasma density much higher than $10^{19} \mathrm{~cm}^{-3}$ then we can not overlook the effect of ionization and electron-ion collisions [18].

\section{Overview on Backward Raman Amplification in Plasma}

The review study of simulation [19-22] and experiment $[23,24]$ results has shown the possibility and potential to focus and compress the intense laser pulses (optical and $\mathrm{X}$-ray regime) through Backward Raman amplification (BRA) in plasmas. We report here the review study of these results and similar scalings for efficient BRA to compress and amplify the intense X-ray pulse. Before explaining the review work we outline first the BRA process in plasmas. BRA is based on the fact that a plasma can withstand very high energy densities due to its ionized nature. This has motivated research to realize a plasma amplifier to further boost the power of an existing CPA system [25] or as an alternative technique that will replace CPA itself [26]. The idea of the Raman effect in various media to amplify laser pulses extends back to work carried out 40 years ago when researchers used gases and liquids to amplify excimer laser pulses for the purpose of laser-based nuclear fusion [27]. The idea of using plasmas for this same purpose is however more recent. Proof-of-principle experiments demonstrating BRA in a plasma have been reported by several groups over the last 10 years. These studies have focused primarily on the amplification of ultrashort Ti:Sapphire laser pulses with the goal of creating ultrahigh peak intensities by significantly increasing the amount of energy contained in a single femtosecond-scale pulse.

BRA in plasmas is a three wave interaction process of laser light with a plasma of density lower than one quarter of the critical density. Here critical density is that density where the plasma frequency equals the laser frequency. The three waves in BRA consist of a long low intensity pump laser pulse, a short low intensity seed laser pulse that is to be amplified, and an electron plasma wave that is excited by the beating of the two laser pulses. The pump and seed are both electromagnetic waves while the electron plasma wave, or Langmuir wave, is an electrostatic wave. The stimulating Raman scattering (SRS) process is somewhat different from the BRA process. The SRS instability occurs when a light wave with a frequency $\omega_{0}$ and wave vector $k_{0}$ enters a plasma and Thomson scatters from noise density fluctuations via a resonant interaction that picks a specific $\omega_{1}$ and $k_{1}$ for the scattered light to conserve energy and momentum at frequency $\omega_{2}=\omega_{0}-\omega_{1}$ and wave vector $k_{2}=k_{0}-k_{1}$. The scattered light in turn beats with the incident light to create a ponderomotive force proportional to the gradient of the product of their individual amplitudes. This force will then reinforce the density noise resulting in even larger perturbations inside the plasma which become a plasma wave with $\omega_{2}$ and $k_{2}$. The plasma wave behaves like a density grating which causes further collective scattering of the incident light as the instability cycle continues. The cycle is maintained as long as phase-matching and conservation of total wave action are satisfied. BRA relies upon the backward SRS instability to amplify the short seed laser pulse. In this direct backscatter geometry the pump laser is collided with a counter-propagating injected short seed laser inside the plasma. The seed laser frequency is downshifted from the pump laser frequency by the plasma frequency in order to achieve the resonance that would have automatically occurred in backward SRS from the plasma noise. Because the injected seed laser in BRA is stronger than the initially Thomson scattered light from backward SRS, the ponderomotive force and the driven plasma wave amplitude in BRA are larger which can induce a greater amount of energy transfer from the pump to the seed mediated by the plasma wave. The seed can cause pump depletion, suppressing the SRS instability. The slow moving plasma wave has a phase velocity in the same direction as the pump, but its group velocity moves with the short laser pulse being amplified. Since this process is based on the backward SRS instability, the seed gets amplified until the pump begins to be depleted.

As mentioned above, the attraction of this idea is due to the fact that a plasma is capable of withstanding laser pulses of very high intensities that normally would destroy solid state optics such as diffraction gratings used in a CPA system. However a plasma exhibits an abundance of other complex nonlinear behavior not found in ordinary media. For instance, a plasma is highly susceptible to additional transverse and longitudinal instabilities in the presence of a high intensity laser beam. The transverse instabilities can adversely affect the laser spot quality and the longitudinal instabilities can cause the pulse shape to break apart. Both types of instabilities can play a role in reducing the efficiency of the energy trans- 
fer process. Therefore the physical restrictions on energy transfer efficiency may place limitations on the amount of seed amplification and the final intensity the seed pulse can reach.

BRA in plasma is a three wave interaction process and can be numerically modeled by fluid model [28]. The interactions among the pump pulse, seed pulse and Langmuir wave are modeled through the Maxwell equations and the electrom momentum and continuity equations. The three wave interaction in plasma can be expressed as,

$$
\begin{aligned}
& \left(\frac{\partial}{\partial t}+v_{1} \frac{\partial}{\partial x}+v_{1}\right) A_{1}=-i c_{1} A_{2} A_{3}, \\
& \left(\frac{\partial}{\partial t}+v_{2} \frac{\partial}{\partial x}+v_{2}\right) A_{2}=-i c_{2} A_{1} A_{3}^{*}, \\
& \left(\frac{\partial}{\partial t}+v_{3} \frac{\partial}{\partial x}+v_{3}\right) A_{3}=-i c_{3} A_{1} A_{2}^{*},
\end{aligned}
$$

where $A_{i}=e E_{i} / m_{e} \omega_{i} c$ is the electric field amplitude, $E_{i}$ of the pump $(i=1)$ and the seed $(i=2)$ pulse normalized by $m_{e} \omega_{i} c / e, m_{e}$ is the electron mass, $\omega_{i}$ is the pulse frequency, $c$ is the speed of light, $v_{i}$ is the group velocity of the light (plasmon) scaled by $c, v_{i}$ is the inverse bremsstrahlung rate, $c_{i}=\omega_{p e}^{2} / 2 \omega_{i}$, and $k_{i}$ is the wave number of the pulses; $A_{3}=\tilde{n}_{e} / n_{e}$ is the Langmuir wave amplitude, $v_{3}$ is the plasmon decay rate, and $c_{3}=(c q)^{2} / 2 \omega_{3}$, where $\omega_{3}=\omega_{p e}=\sqrt{4 \pi n_{e}^{2} / m_{e}}$ and $q$ is the Langmuir wave number. The energy conservation in BRA reads $\omega_{1}=\omega_{2}+\omega_{p e}$ and the momentum conservation reads $q=k_{1}+k_{2}$.

\section{Recent Work and Scalings on BRA}

Past work on BRA in optical regime has shown the applicability of this novel process in future laser system as a plasma amplifier and compressor. The findings of BRA in optical regime has motivated the several group to implement the BRA process in X-ray regime. We focus here on reviewing the results based on focusing and compression of intense X-ray laser pulse in plasmas via the BRA process. Compression of powerful X-ray pulses to attosecond durations by stimulating Raman backscattering in plasmas has been reported by Malkin et al. [18]. They estimated the theoretical short wavelength limit for intense X-ray pulse compression and focusing through BRA in plasma medium. They reported the $1 \mathrm{~nm}$ shortest wavelength $\mathrm{mJ} X$-ray pulse that can be compressed and focussed to intensities sufficient for vacuum breakdwon intensity $10^{29} \mathrm{~W} / \mathrm{cm}^{2}$. To attain these high intensites, however needs the nonlinear suppression of Landay damping of the Langmuir waves mediating the energy transfer from the pump to the seed pulse. Malkin and Fisch [29] further classified the quasitransient regime of backward Raman amplification of intense X-ray laser pulses. Quasitransient regime are of the most critical importance for X-rays BRA because the X-ray BRA needs plasma densites as large as those of condensed matter in order to provide enough coupling between the pump and seed laser pulse. High density plasma causes strong damping of the Langmur wave that mediatee energy transfer from pump to seed pulse. Such strong damping could reduce the coupling efficiency and BRA will be hard to detect. Since the assumption that the linear BRA is transient (i.e. that the Langmuir wave damping can be neglected within the pumped pulse duration) could be justified only for a small enough Langmuir wave damping, while the stronger damping would make BRA harder to observe. In summary the efficient QBRA is capable of tolerating the mediating Langmuir wave damping exceeding the linear Raman growth rate up to 20 times for strong seed pulse, and up to 10 times for moderate seed pulses. Malkin and Fisch [30] investigated further the plasma parametric regime, where the efficient QBRA of powerful X-ray laser pulse is possible in dense plasma with multicharged ions. Their theoretical calculation is applicable to infrared, ultraviolet, soft X-ray and $\mathrm{X}$-ray laser pulse. They demonstrated the electron temperature-concentration regime for effecient QBRA of Xray at $\lambda=10 \mathrm{~nm}$ and $1 \mathrm{~nm}$. At this wavelength regime the required electron plasma concentration needed are comparable to the compressed condensed matter. In this wavelength regime, the large ion charge can no longer be tolerated.

The work by R. Trines et al. [22] at Rutherford Appleton Laboratory identified the parametric regime for the BRA in which a $4 \mathrm{TW}, 700 \mu \mathrm{m}$ full-width at halfmaximum (FWHM), 25 ps long laser with $800 \mathrm{~nm}$ wavelength can be amplified to $2 \mathrm{PW}$ peak intensity with 35 efficiency. In addition they shown that the same process can be scaled appropriately to compress a 250 fs long, $0.2 \mu \mathrm{m}$ wide soft X-ray pulse (10 $\mathrm{nm}$ wavelength),as produced by facilities like FLASH or LCLS, to subfemtosecond duration and $500 \mathrm{TW}$ peak power, that is, $10^{21} \mathrm{~W} / \mathrm{cm}^{2}$. They argued that seed pulses having very small spot sizes may be affected by various transverse effects such as self-focusing and filamentation. They emphasized the importance of finding the right combination of plasma density, pump intensity, and propagation distance for the purpose of maintaining the focusability of the wide seed and keeping a very low level of filamentation so high peak intensities can be reached. The optimal regime for efficient Raman amplification (as shown by Trines et al.) is estimated by incorporating the effect of the plasma density and the pump intensity on energy-transfer efficiency (determined by the pump Raman backscattering growth rate) and instabilities (determined by the growth rates of pump Raman forward scat- 
terin and probe filamentation).

We followed the optimal regime for efficient BRA as obtained by Trines et al. [22] to realize the BRA in X-ray regime while keeping the ratio $\omega_{0} / \omega_{p}$ in the range of $14-20$ and the normalised laser field

$a_{0}=0.85 \times 10^{-9} \sqrt{I\left(\mathrm{~W} / \mathrm{cm}^{2}\right)} \lambda(\mu \mathrm{m})$ in the range of 0.01 -0.03 . We have shown below the window of plasma density and pump laser intensity corresponding to pump laser wavelngth. We may expect the efficient BRA in $\mathrm{X}$-ray regime for the given optimal value of laser-plasma parameters and can be tested via the PIC simulation but needs high accuracy on account of time and efficinet computing facility.

Scanning of the parameters (i.e. laser intensity and plasma density as mentioned in Table 1) reflects the fact that amplification of 1 angstrom X-ray laser pulse needs the plasma density $10^{26} \mathrm{~cm}^{-3}$ (available at NIF facility) and corresponding pump FWHM intensity should be order of $10^{22} \mathrm{~W} / \mathrm{cm}^{2}$ for efficient BRA. The parametric regime needed for efficient BRA is still looking far away for practical realization of XFEL pulse amplification. Since the efficient operation of the process needs the joint facility of NIF and LCLS. Hence due to unavailability of practical tools, we may test the efficient BRA $\mathrm{X}$-regime via the simulation codes. However we need to modify our existing Particle-In-Cell (PIC) simulation codes to test BRA in $\mathrm{x}$-regime where we expect to have very high plasma density and very intense pump FWHM field. Since most of PIC codes are considering the collisionless plasma but in high density plasma, we can not ignore the role of electron-ion collisions and we need to implement the collisional process (including absorption of laser energy and Landau damping [18]) in simulation code.

To practise the BRA in X-regime we also need to modify PIC code at high laser intensity by modifying the exact force experienced by the particles in plasma medium. Since the laser-plasma interaction at extremely high laser intensities, elctrons can become ultrarelativistic within a fraction of wave period experiencing superstrong accelerations and therefore emitting relativily large amount of electromagnetic radiation. Radiation Reaction (RR) is the

Table 1. Summary of parameters for efficient BRA in X-ray regime.

\begin{tabular}{ccc}
\hline$\lambda(\mathrm{nm})$ & $\begin{array}{c}\text { Electron density } \\
\left(\mathrm{cm}^{-3}\right)\end{array}$ & $\begin{array}{c}\text { Pump FWHM intensity } \\
\left(\mathrm{W} / \mathrm{cm}^{2}\right)\end{array}$ \\
\hline 10 & $1 \times 10^{22}$ & $1 \times 10^{18}$ \\
1 & $1 \times 10^{24}$ & $1 \times 10^{20}$ \\
0.1 & $1 \times 10^{26}$ & $1 \times 10^{22}$ \\
0.01 & $1 \times 10^{28}$ & $1 \times 10^{24}$ \\
\hline
\end{tabular}

influence of electromagnetic field emitted by each electron on the motion of electron itself [31] and may become essential under extreme conditions mentioned above. Recently it is found [32] that at intensities exceeding $10^{22} \mathrm{~W} / \mathrm{cm}^{-2}$ the RR force strongly affects the dynamics for a linearly polarized laser pulse. There is need to test the BRA operation of X-ray laser pulse in PIC simulations with RR effects included either using an approach similar to the Landau-Lifshitz (LL) equation [31] or using a different RR modeling [33].

\section{Possibility of Focusing X-Ray Beam Using Plasma Waveguide}

The plasma channel having a parabolic profile of the electron density with a density minimum along the axis acts as an optical waveguide. The optical guiding in plasma may be of practical interest to focus [34] the x-ray beams. Kukhlevsky and Kozma [35] investigated the modal and focusing properties of the plasma based waveguide having a parabolic profile of the electron density. They pointed out that the focusing properties of plasma waveguide structure depend on waveguide length and wavelength. To focus the parallel soft X-ray beam $(\lambda=40 \mathrm{~nm})$ in 1-cm-long plasma guide they calculated the plasma density gradient $10^{23} \mathrm{~cm}^{-5}$. However their model to focus the $\mathrm{x}$-ray beam is based on paraxialenvelope equations that only considers the central portion of the beam (portion of the beam near to axis), not the whole beam. We may further extend this model considering the non-paraxial approach where one may include the on axis and off axis portion of the beam in the model calculations. The non-paraxial model of plasma waveguide may predict more accurate results in favor of X-ray beam focusing through plasma wave-guide.

\section{REFERENCES}

[1] W. Ackermann, et al., "Operation of a Free-Electron Laser from the Extreme Ultraviolet to the Water Window," Nature Photonics, Vol. 1, No. 6, 2007, pp. 336-342. doi:10.1038/nphoton.2007.76

[2] P. Emma, et al., "First Lasing and Operation of an Angstrom-Wavelength Free-Electron Laser," Nature Photonics, Vol. 4, No. 9, 2010, pp. 641-647. doi:10.1038/nphoton.2010.176

[3] T. Ishikawa, et al., "A Compact X-Ray Free-Electron Laser Emitting in the Sub-Ångström Region," Nature Photonics, Vol. 6, No. 11, 2012, pp. 540-544. doi:10.1038/nphoton.2012.141

[4] L. Young, et al., "Femtosecond Electronic Response of Atoms to Ultra-Intense X-Rays," Nature, Vol. 466, No. 7302, 2010, pp. 56-61. doi:10.1038/nature09177

[5] G. Doumy, et al., "Nonlinear Atomic Response to Intense Ultrashort X-Rays," Physical Review Letters, Vol. 106, No. 8, 2011, Article ID: 083002. 
doi:10.1103/PhysRevLett.106.083002

[6] M. Hoener, et al., "Ultraintense X-Ray Induced Ionization, Dissociation, and Frustrated Absorption in Molecular Nitrogen," Physical Review Letters, Vol. 104, No. 25, 2010, Article ID: 253002. doi:10.1103/PhysRevLett.104.253002

[7] J. P. Cryan, et al., "Auger Electron Angular Distribution of Double Core-Hole States in the Molecular Reference Frame," Physical Review Letters, Vol. 105, No. 8, 2010, Article ID: 083004.

doi:10.1103/PhysRevLett.105.083004

[8] L. Fang, et al., "Double Core-Hole Production in $\mathrm{N}_{2}$ : Beating the Auger Clock," Physical Review Letters, Vol. 105, No. 8, 2010, Article ID: 083005. doi:10.1103/PhysRevLett.105.083005

[9] S. M. Vinko, et al., "Creation and Diagnosis of a SolidDensity Plasma with an X-Ray Free-Electron Laser," Nature, Vol. 482, No. 7383, 2012, pp. 59-62. doi:10.1038/nature 10746

[10] Reiche, et al., "Start-to-End Simulation for the LCLS X-Ray FEL," Nuclear Instruments and Methods A, Vol. 483, No. 1, 2002, pp. 70-74. doi:10.1016/S0168-9002(02)00288-7

[11] P. Emma, et al., "Femtosecond and Subfemtosecond XRay Pulses from a Self-Amplified Spontaneous-Emission Based Free-Electron Laser," Physical Review Letters, Vol. 92, No. 7, 2004, Article ID: 074801. doi:10.1103/PhysRevLett.92.074801

[12] E. L. Saldin, et al., "Scheme for Attophysics Experiments at a X-Ray SASE FEL," Optics and Communications, Vol. 212, No. 4, 2002, pp. 377-390. doi:10.1016/S0030-4018(02)02008-4

[13] A. Jarre, et al., "Two-Dimensional Hard X-Ray Beam Compression by Combined Focusing and Waveguide Optics," Physical Review Letters, Vol. 94, No. 7, 2005, Article ID: 074801.

[14] C. G. Schroer, et al., "Hard X-Ray Nanoprobe Based on Refractive X-Ray Lenses," Applied Physics Letters, Vol. 87, No. 12, 2005, Article ID: 124103. doi:10.1063/1.2053350

[15] W. Liu, et al., "Short Focal Length Kirkpatrick-Baez Mirrors for a Hard X-Ray Nanoprobe," Review of Scientific Instruments, Vol. 76, No. 11, 2005, Article ID: 113701. doi:10.1063/1.2125730

[16] C. Bergemann, et al., "Focusing X-Ray Beams to Nanometer Dimensions," Physical Review Letters, Vol. 91, No. 20, 2003, Article ID: 204801. doi:10.1103/PhysRevLett.91.204801

[17] C. G. Schroer, et al., "Focusing Hard X Rays to Nanometer Dimensions by Adiabatically Focusing Lenses," Physical Review Letters, Vol. 94, No. 5, 2005, Article ID: 054802. doi:10.1103/PhysRevLett.94.054802

[18] V. M. Malkin, et al., "Compression of Powerful X-Ray Pulses to Attosecond Durations by Stimulated Raman Backscattering in Plasmas," Physical Review E, Vol. 75, No. 2, 2007, Article ID: 026404. doi:10.1103/PhysRevE.75.026404

[19] P. J. Mardahl, et al., "Intense Laser Pulse Amplification
Using Raman Backscatter in Plasma Channels," Physics Letters A, Vol. 296, No. 2, 2002, pp. 109-116. doi:10.1016/S0375-9601(02)00194-9

[20] D. S. Clark and N. J. Fisch, "Particle-in-Cell Simulations of Raman Laser Amplification in Preformed Plasmas," Physics of Plasmas, Vol. 10, No. 12, 2003, pp. 4848-4855. doi: $10.1063 / 1.1625940$

[21] D. S. Clark and N. J. Fisch, "Raman Laser Amplification in Preformed and Ionizing Plasmas," Laser and Particle Beams, Vol. 23, No. 1, 2005, pp. 101-106. doi: $10.1017 / \mathrm{S} 0263034605050172$

[22] R. M. G. M. Trines, et al., "Simulations of Efficient Raman Amplification into the Multipetawatt Regime," $\mathrm{Na}$ ture Physics, Vol. 7, No. 1, 2011, pp. 87-92. doi:10.1038/nphys 1793

[23] W. Cheng, et al., "Reaching the Nonlinear Regime of Raman Amplification of Ultrashort Laser Pulses," Physical Review Letters, Vol. 94, No. 4, 2005, Article ID: 045003. doi:10.1103/PhysRevLett.94.045003

[24] J. Ren, et al., "A Compact Double-Pass Raman Backscattering Amplifier/Compressor," Physics of Plasmas, Vol. 15, No. 5, 2008, Article ID: 056702. doi: $10.1063 / 1.2844352$

[25] D. Strickland and G. Mourou, "Compression of Amplified Chirped Optical Pulses," Optics and Communications, Vol. 56, No. 3, 1985, pp. 219-221. doi:10.1016/0030-4018(85)90120-8

[26] N. J. Fisch and V. M. Malkin, "Generation of Ultrahigh Intensity Laser Pulses," Physics of Plasmas, Vol. 10, No. 5, 2003, pp. 2056-2063. doi:10.1063/1.1567290

[27] J. R. Murray, et al., "Raman Pulse Compression of Excimer Lasers for Application to Laser Fusion," IEEE Journal of Quantum Electronics, Vol. 15, No. 5, 1979, pp. 342-368. doi:10.1109/JQE.1979.1070009

[28] C. J. McKinstrie and A. Simon, "Nonlinear Saturation of the Absolute Stimulated Raman Scattering Instability in a Finite Collisional Plasma," Physics of Fluids, Vol. 29, No. 6, 1986, pp. 1959-1970. doi:10.1063/1.865623

[29] V. M. Malkin and N. J. Fisch, "Quasitransient Regimes of Backward Raman Amplification of Intense X-Ray Pulses," Physical Review E, Vol. 80, No. 4, 2009, Article ID: 046409. doi:10.1103/PhysRevE.80.046409

[30] V. M. Malkin and N. J. Fisch, "Quasitransient Backward Raman Amplification of Powerful Laser Pulses in Dense Plasmas with Multicharged Ions," Physics of Plasmas, Vol. 17, No. 7, 2010, Article ID: 073109. doi:10.1063/1.3460347

[31] L. D. Landau and E. M. Lifshitz, "The Classsical Theory of Fields," 2nd Edition, Elsevier, Oxford, 1975.

[32] M. Tamburini, F. Pegoraro, A. Di Piazza, C. H. Keitel and A. Macchi, "Radiation Reaction Effects on Radiation Pressure Acceleration," New Journal of Physics, Vol. 12, No. 12, 2010, Article ID: 123005. doi:10.1088/1367-2630/12/12/123005

[33] A. Zhidkov, J. Koga, A. Sasaki and M. Uesaka, "Radiation Damping Effects on the Interaction of Ultraintense Laser Pulses with an Overdense Plasma," Physical Review Letters, Vol. 88, No. 18, 2002, Article ID: 185002. 
doi:10.1103/PhysRevLett.88.185002

[34] N. H. Burnett and P. B. Corkum, "Cold-Plasma Production for Recombination Extremeultraviolet Lasers by Optical-Field-Induced Ionization," Journal of Optical Society of America B, Vol. 6, No. 6, 1989, pp. 1195-1199.
doi:10.1364/JOSAB.6.001195

[35] S. V. Kukhlevsky and L. Kozma, "Modal and Focusing Properties of Plasma Based Waveguides for X-Ray Beams," Contributions to Plasma Physics, Vol. 38, No. 5, 1998, pp. 583-597. doi:10.1002/ctpp.2150380503 